\title{
Comparison of temperature dynamics of various thermal cycler calibration methods
}

\author{
Mary $\operatorname{Span}^{1, *}$, Marc Verblakt ${ }^{2}$, and Tom Hendrikx ${ }^{1}$ \\ ${ }^{1}$ CYCLERtest BV, Rötscherweg 61, 6374 XW Landgraaf, the Netherlands \\ ${ }^{2}$ CelsiusLabs, Rötscherweg 61, 6374 XW Landgraaf, the Netherlands
}

\begin{abstract}
A multitude of testing laboratories, including medical, forensic, food, water and environmental testing laboratories, use thermal cycler equipment for Polymerase Chain Reaction (PCR) based DNA testing. PCR reactions are temperature critical and therefore these thermal cyclers require regular calibration to ensure correct functioning. Over time, a variety of calibration and verification methods have been published. Most of these methods are not traceable to the International Systems of Units (SI) and are connected to substantial measurement uncertainties, and therefore inapt for application in metrology. Physical temperature sensor based methods have found to be the most appropriate methods. These temperature sensor based methods can be categorized in in-tube and in-well methods. The in-tube methods measure temperature inside the PCR reaction tubes, which are placed in the wells of the thermal cycler heating block. The in-well methods measure temperature directly in the wells of the thermal cycler heating block. Both in-tube and in-well methods can be designed to be metrological traceable. However, the associated measurement uncertainty can vary substantially between in-tube and in-well methods. This paper compares the temperature dynamics of in-tube and in-well methods and discusses systematic and random errors which can contribute to the measurement uncertainty of both methods.
\end{abstract}

\section{Introduction}

The number of testing laboratories requiring calibration of their thermal cyclers has strongly increased over the past years, due to their need to meet the requirements of the ISO 15189 and ISO/IEC 17025. Therefore the necessity for an appropriate calibration method for thermal cyclers has also increased. The calibration method should be traceable to SI and be associated with a low measurement uncertainty to be suitable for application in metrology.

An earlier study [1] has shown that biochemistry or chemistry based methods and non-contact physical methods are inapt for application in metrology due to lacking traceability to SI and/or large associated measurement uncertainties. Temperature sensor based physical methods have shown to be the most suitable methods for metrology. These temperature sensor based physical methods can be categorized in in-tube methods $[2,3,4,5,6]$ and in-well methods [7, $8,9,10,11,12,13]$. The in-tube methods have been developed from the molecular biologist perspective, in order to mimic the PCR reaction as close as possible. The in-well methods have been developed from the metrologist perspective, in order to be able to measure temperatures with the lowest possible measurement uncertainty. As most intube methods have been developed by non-metrologists, little attention has been paid to measurement uncertainty during the method development. This has resulted in in-tube methods with large measurement uncertainties due to both systematic and random errors [1].

To be able to compare the temperature dynamics of in-tube and in-well methods the published in-tube methods have been improved to avoid systematic errors and reduce the measurement uncertainty. The goal was to develop a technically outstanding in-tube method, which was based on the same temperature sensors and measurement hardware as the reference in-well method [13], with a minimal measurement uncertainty. This optimal in-tube method allowed comparing the temperature dynamics between in-well and in-tube methods without other perturbing influences apart from the differences in sensor construction.

\section{Development in-tube method}

The in-tube method has been developed in several steps, taking into account the flaws of published methods as described by Span et al. [1]. During the development both static and dynamic temperature measurements where performed. Temperature accuracy and response time measurements were performed by positioning the in-tube sensor construction in a GENOtronics temperature calibration block with 24 ITS-90 traceable reference temperature sensors

Corresponding author: marys@,cyclertest.com

(C) The Authors, published by EDP Sciences. This is an open access article distributed under the terms of the Creative Commons Attribution License 4.0 
(GENOtronics, the Netherlands). Dynamic temperature measurements were performed by positioning the in-tube sensor construction in a MJ Research PTC-200 thermal cycler (MJ Research, USA) or Corbett Research PalmCycler (Corbett Research, Australia). In-tube measurements were performed without thermal contact medium between tube and well and with the heated lid closed, as this represents the normal use of tubes in a thermal cycler.

Starting point was the in-tube sensor construction as published by Yang et al. [2]. For the initial in-tube sensor construction Extreme Uniform $0.2 \mathrm{ml}$ thin-wall tubes (BIOplastics, the Netherlands) were used. These tubes have been selected based on their tight tube-to-well fit, their thin and uniform walls and low evaporation rates. The $0.2 \mathrm{ml}$ tubes were filled with $25 \mathrm{ul} \mathrm{PCR}$ mix and thermistors with a diameter of $0.8 \mathrm{~mm}$ and $0.1 \mathrm{~mm}$ insulated lead wires were placed in the tubes, without fixation. The thermistors without tubes were calibrated traceable to international standards (International Temperature Scale 1990) with an expanded combined measurement uncertainty of $0.02{ }^{\circ} \mathrm{C}$. The holes in the caps, through which the sensor wires were led, were sealed with epoxy resin to reduce evaporation to a minimum. Modifications to this initial in-tube sensor construction were done step by step to enable evaluation of the impact of each modification.

The initial measurements showed mass loss due to evaporation. The evaporation rates were up to $5 \%$ during the $60 \mathrm{~s}$ $20{ }^{\circ} \mathrm{C}, 900 \mathrm{~s} 95{ }^{\circ} \mathrm{C}, 900 \mathrm{~s} 20{ }^{\circ} \mathrm{C}$ protocol. If this in-tube sensor construction would be used repeatedly the lost mass would increase with every measurement. Therefore, the PCR mix was replaced by synthetic oil to eliminate mass loss of the in-tube sensor construction due to evaporation. As the heat capacity of oil is lower than the heat capacity of PCR mix, 54 ul oil was required to equal the heat capacity of 25 ul PCR mix. The in-tube sensor construction with oil showed no weight loss due to evaporation. However, this in-tube sensor construction showed substantial temperature variation from sensor to sensor, more than what was expected based on variability between thermistors from one production batch. The cause was found in the positioning of the sensor. In the tubes where the higher temperatures were reported the sensor lay against the tube wall, rather than being positioned freely in oil.

In a following step, the thermistor was centred in the tube with a rigid plastic sleeve. This strongly reduced the variation between sensors. However, during the first measurements in a thermal cycler with the heated lid closed, temperatures were measured higher than the reference in-well method. This proved to be due to the heat transfer from the heated lid through the sensor wires.

In the final step, the thermistor was thermally anchored to reduce heat loss via sensor wires to the environment and heat transfer via sensor wires from the heated lid of the thermal cycler. Only after this fourth improvement the in-tube sensor construction was capable of measuring temperatures without systematic errors and minimal random errors. The difference in measured temperature between de thermally anchored and non-anchored thermistor was $0.04{ }^{\circ} \mathrm{C}$ (Figure 1).

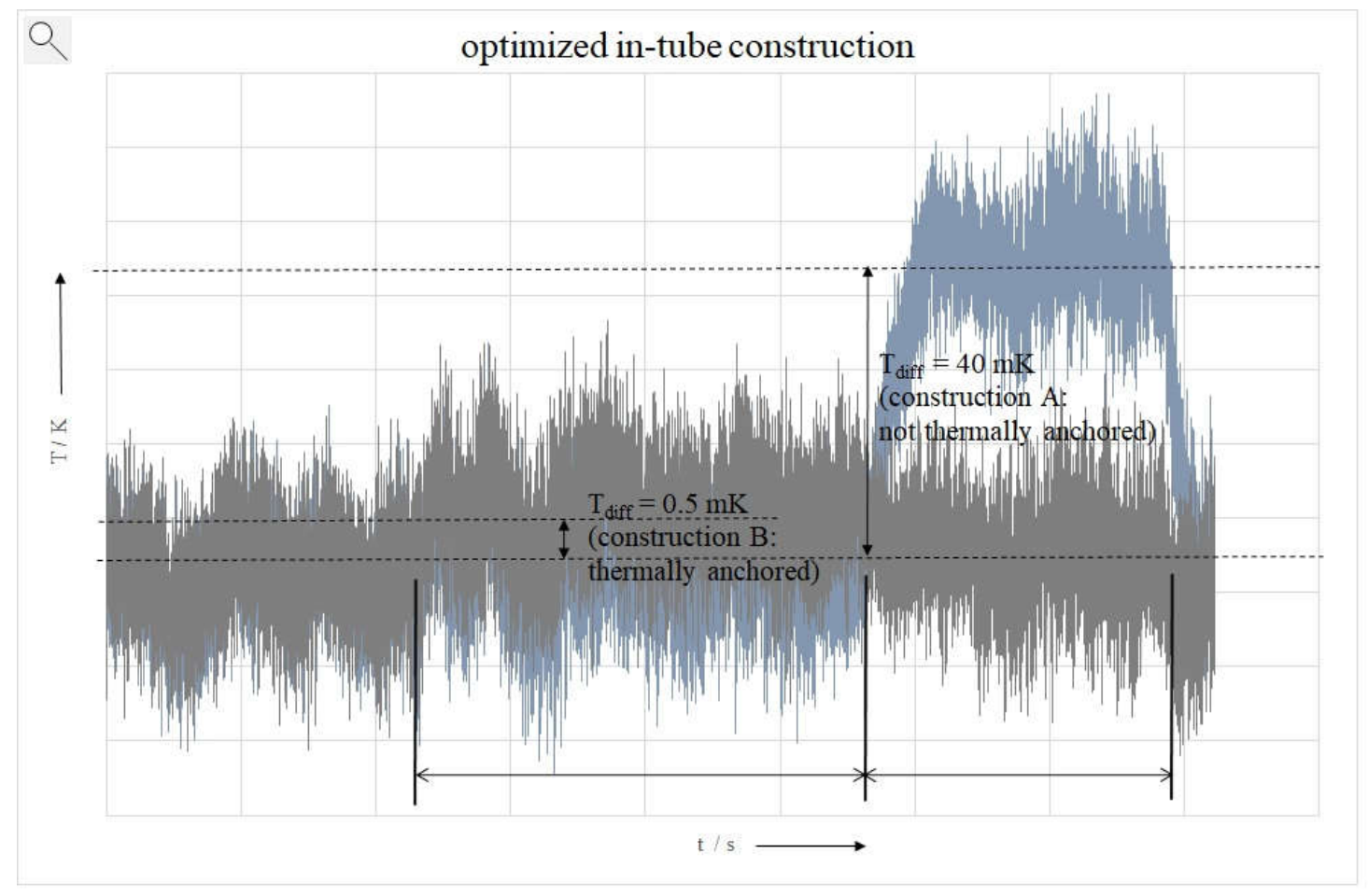

Fig. 1. Effect of thermal anchoring of thermistor in in-tube sensor construction. The non-anchored thermistor reports a $0.04{ }^{\circ} \mathrm{C}$ higher temperature in comparison to the thermally anchored thermistor due to heating of the sensor wires by the heated lid of the thermal cycler. 


\section{In-tube versus in-well method}

Before comparing the in-tube and in-well method in a thermal cycler, the response times (Tau times) were determined for the in-tube sensor construction in PCR mix and oil and the in-well sensor construction by preconditioning the sensors in a calibration or thermal cycler block at $23{ }^{\circ} \mathrm{C}$ and then transferring them to a calibration or thermal cycler block set at $90^{\circ} \mathrm{C}$ or $95^{\circ} \mathrm{C}$. The sampling frequency was $8 \mathrm{~Hz}$. The response times were respectively $6,6 \mathrm{~s}, 7,9 \mathrm{~s}$ and 2,0 $\mathrm{s}$ for the in-tube method with PCR mix, the in-tube method with oil and the in-well method (see figure 2).

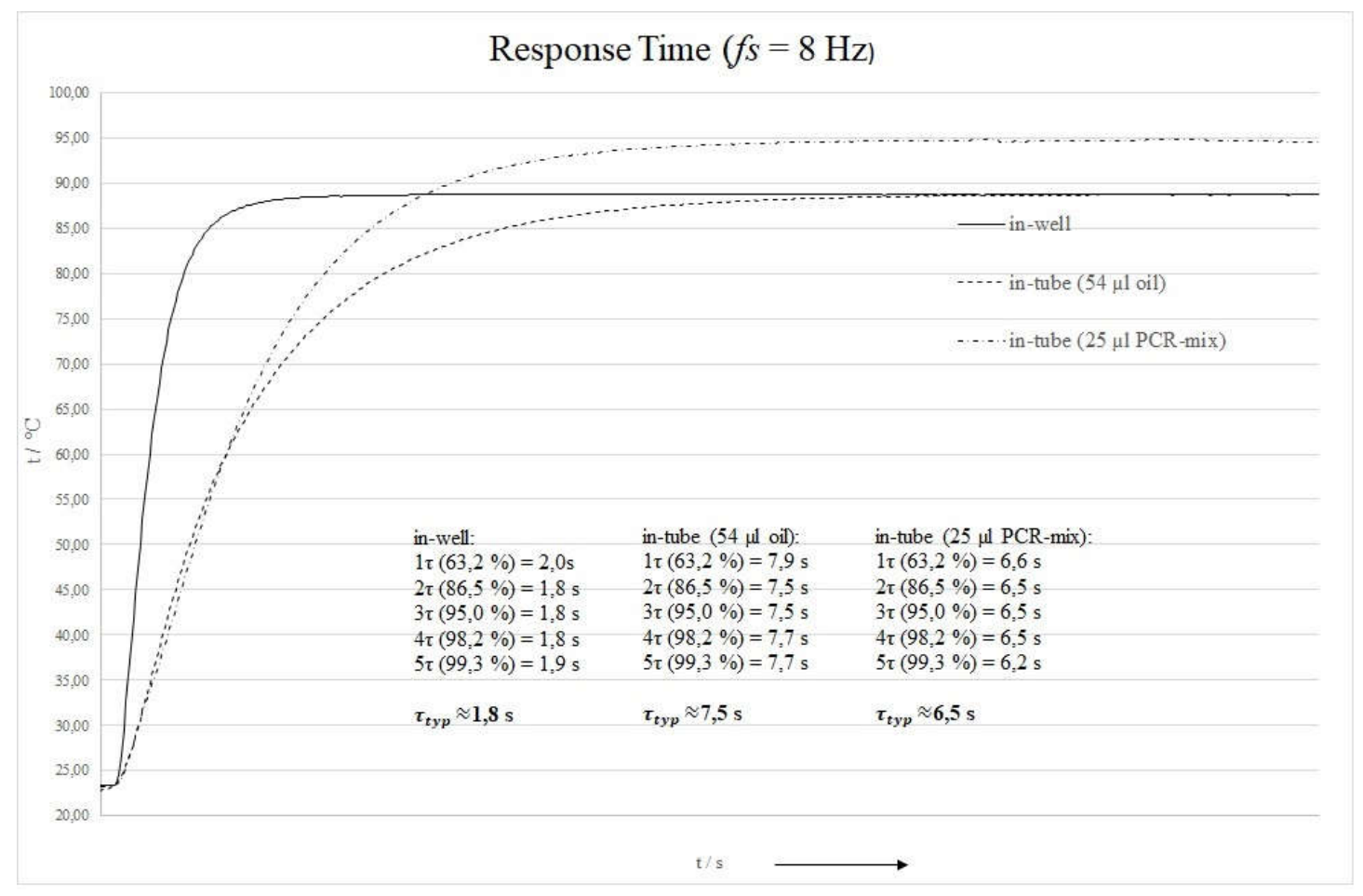

Fig. 2. Response times of the different in-tube and in-well sensor constructions.

For the comparison of the temperature dynamics of the in-tube and in-well method, the in-well method of the MTAS system [13] was used as reference. The MTAS system is a commercially available metrological traceable inwell method using 15 thermistors with a combined expanded uncertainty of $0.03{ }^{\circ} \mathrm{C}$ (CYCLERtest, the Netherlands). All in-tube measurements were performed without thermal contact medium between well and tube to be representative of the normal use in a thermal cycler. All measurements were performed with the heated lid closed, to be representative of the normal use during a PCR reaction. Temperature data were collected with a sampling frequency of $8 \mathrm{~Hz}$. The dynamic temperature measurements where performed with the following protocol: $60 \mathrm{~s} 20{ }^{\circ} \mathrm{C}, 900 \mathrm{~s} 95^{\circ} \mathrm{C}, 900 \mathrm{~s} 20{ }^{\circ} \mathrm{C}$. The thermal cycler was programmed at maximum heat rate.

The dynamic temperature measurements showed that the measured temperatures in the static parts of the curves differed as little as $-0.06{ }^{\circ} \mathrm{C}$ at $95{ }^{\circ} \mathrm{C}$ after $60 \mathrm{~s}$ stabilisation between the in-tube and in-well methods (see figure 3 ). This difference is close to the measurement uncertainties of both the in-tube and in-well method. When the measurement was performed with the heated lid opened, a difference of $-0.5 \mathrm{C}$ was observed. So the in-well temperature can only be approached when closing the heated lid.

The major differences between the in-tube and in-well method were observed in the dynamic part of the curve (see figure 3). The in-tube sensor construction lags behind the in-well sensor construction, although reported heat rates do not differ significantly. The lag time is directly proportional to the difference in response times of the sensor constructions. Furthermore, the in-tube sensor construction shows no overshoot, whereas the in-well sensor construction does. This effect is related to the difference in the response times of the in-tube and in-well sensor construction, but also to the difference in thermal diffusivity of the different materials used in the sensor constructions. The heat diffusivity of the copper used in the in-well sensor construction is more than a factor 1000 higher than the heat diffusivity of oil used in the in-tube sensor construction. The heat diffusivity of water and therefore also PCR mix as about a factor 2 higher than the heat diffusivity of oil. As the in-tube method should mimic the PCR reaction as close as possible, it is not desirable to correct for this difference in thermal diffusivity between water or oil and copper.

The selected $0.2 \mathrm{ml}$ tube (BIOplastics, the Netherlands) had an optimal fit to the well, as had been checked upfront in a tube-to-well fit test. The contact error of the in-tube method with oil was determined to be $0.05{ }^{\circ} \mathrm{C}$ and therefore had a limited impact on the measured static values and mainly contributed to the measurement uncertainty. The expanded combined measurement uncertainty of the in-tube method was calculated to be $0.06{ }^{\circ} \mathrm{C}(k=2)$. Therefore the $0.06^{\circ} \mathrm{C}$ difference between the in-tube and in-well methods is within the limits of the measurement uncertainty. 

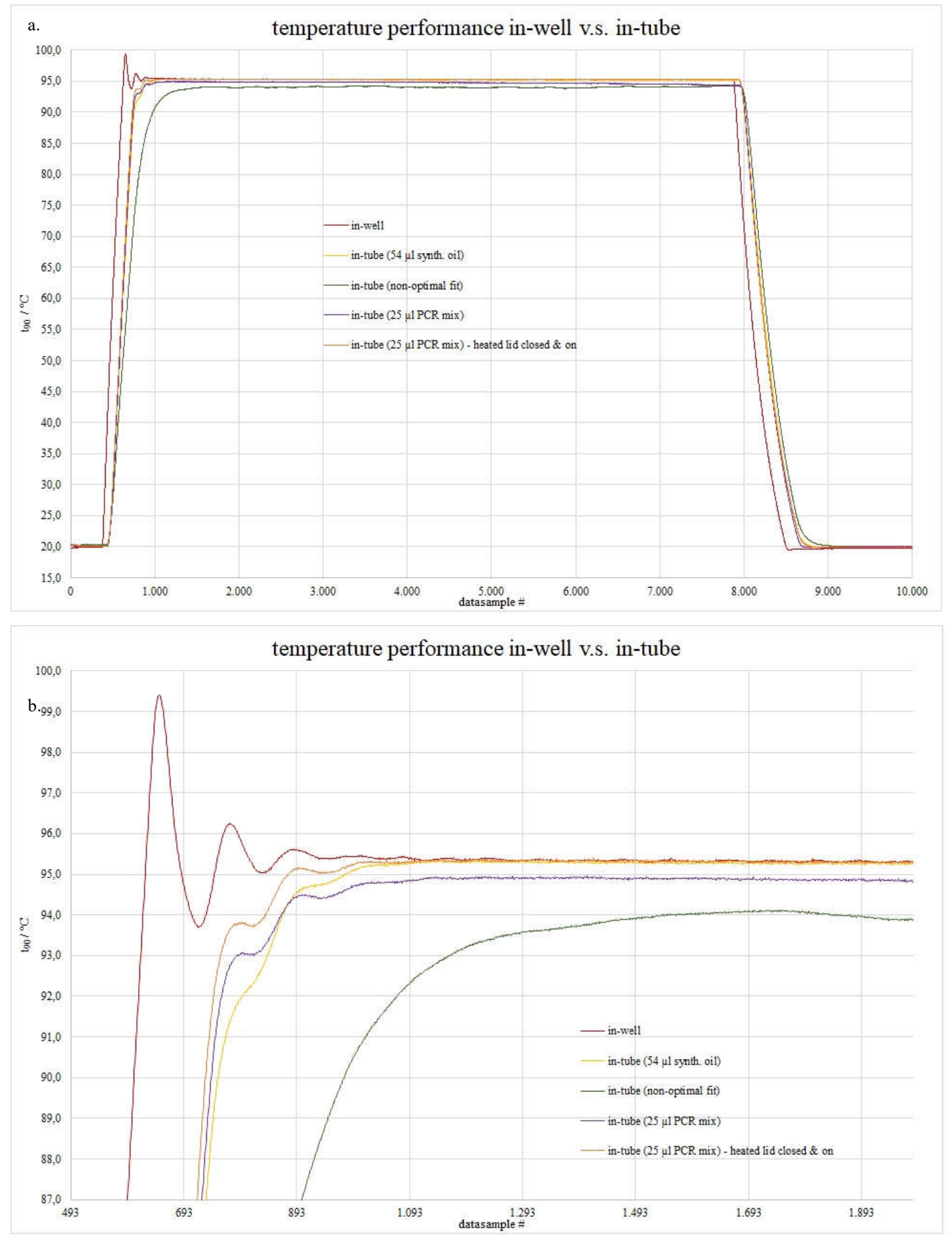

Fig. 3. Temperature performance of thermal cycler measured with several in-tube and in-well sensor constructions in overview graph (a) and zoomed-in graph (b).

As the contact error was found to be the largest contribution in the uncertainty of the in-tube method it became clear that the tube-to-well fit was crucial for in-tube methods. A number of other $0.2 \mathrm{ml}$ tubes with a poor tube-to-well fit have also been tested. The poor tube-to-well fit resulted in a significant difference in measured temperatures. More time was required to reach equilibrium and after 15 minutes stabilisation the temperature was still $1.5^{\circ} \mathrm{C}$ lower (see figure 3). So $0.2 \mathrm{ml}$ tubes with a poor tube-to-well fit resulted in a systematic error of $1.5^{\circ} \mathrm{C}$ in the static part of the curve, which resulted in an expanded combined measurement uncertainty of $1.73{ }^{\circ} \mathrm{C}(k=2)$. In the dynamic part of the curve substantial lagging of the measured temperature is observed (see figure 3 ). 


\section{Conclusions}

Comparison of the temperature dynamics of in-tube and in-well thermal cycler calibration methods shows that it is feasible to use an in-tube method to calibrate thermal cyclers. However, care needs to be taken in the design of the intube methods to avoid systematic errors. This study has shown that the following aspects are essential for in-tube methods without systematic errors and a low measurement uncertainties: (1) thermal anchoring of thermistors to eliminate the stem-effect and inverse stem-effect (2) use of oil instead PCR mix to prevent evaporation, (3) optimal tube-to-well fit to minimize the contact error, (4) measuring with the heated lid closed to guarantee an optimal tube-towell contact through the heated lid pressure, and (5) to be representative of the normal use of tubes in thermal cyclers the measurement should be performed without contact medium and with the heated lid closed and turned on to achieve the same temperature as in normal use. When all these aspects are taken into account expanded combined measurement uncertainties as low as $0.06{ }^{\circ} \mathrm{C}(k=2)$ can be achieved with an in-tube method versus $0.03{ }^{\circ} \mathrm{C}(k=2)$ with an in-well method. The major contribution to this difference in measurement uncertainty is the contact error of the in-tube method, which was found to be $0.05{ }^{\circ} \mathrm{C}$.

After $60 \mathrm{~s}$ stabilisation time the in-tube and in-well method report temperatures that differ less than the measurement uncertainty of the in-tube method. Before $60 \mathrm{~s}$ stabilisation time the in-tube and in-well method show differences which can be explained by differences in response times due to the differences in sensor constructions and thermal diffusivity of the materials used in the sensor constructions.

When using tubes with a suboptimal tube-to-well fit the contact error increases above $1{ }^{\circ} \mathrm{C}$ and will result in an expanded combined measurement uncertainty exceeding $1{ }^{\circ} \mathrm{C}(k=2)$. Although the described optimal in-tube method performs well, under the conditions as described, it is not practical for routine use in metrology and calibration. When changing tubes or thermal cyclers, the contact error would have to be re-assessed per measurement and the combined expanded measurement uncertainty would have to be re-calculated for each measurement, as it is strongly dependent on the contact error. This is cumbersome and therefore impractical. Furthermore, if the combined expanded measurement uncertainty exceeds $0,15^{\circ} \mathrm{C}$ it does not allow meaningful compliancy testing for thermal cyclers, as in PCR deviations as low as $0.5^{\circ} \mathrm{C}$ can already result in different, incorrect or failing PCR results.

So overall, it is feasible to design in-tube methods with expanded combined measurement uncertainties as low as $0.06{ }^{\circ} \mathrm{C}(k=2)$. However, as the measurement uncertainty is strongly dependent on the contact error, which can strongly vary between measurements, in-tube methods are not practical for routine use in metrology.

\section{References}

1. M. Span, M. Verblakt, T. Hendrix https://cfmetrologie.edpsciences.org/articles/metrology/pdf/2017/01/metrology metr2017 05003.pdf

2. I. Yang, Y.-H. Kim, J.-Y. Byun, S.-R. Park, Analytical Biochemistry, 338, 192-200 (2005)

3. Schoder, 2005, J Clin Microbiology, 43:6, 2724-2728 (2005)

4. Y.-H. Kim, I. Yang, Y-S. Bae, S.-R. Park, BioTechniques, 44, 495-505 (2008)

5. D. Schoder, A. Schmalwieser, G. Schauberger, K. Kuhn, J. Hoorfar, M. Wagner, Clin Chem, 49:6, 960-963 (2003)

6. Thermal cycler calibration service http://www.totalq.gr/en/services/calibrations/temperature-relative-humidity.html

7. On-site Temperature Verification Service https://www.thermofisher.com/nl/en/home/products-andservices/services/instrument-qualification-services/compliance-and-validation/on-site-temperature-verification.html

8. On-site thermal cycler temperature calibration service http://www.lbt-blt.be/nl/etalonage/validation.php

9. On-site thermal cycler temperature calibration service http://www.idoslab.com/servicios.html

10. PCR cycler service https://nl.vwr.com/store/content/externalContentPage.jsp;jsessionid=ps2jvBDF2b7j38L6n2wmqmL6.estore5b?path $=/$ nl.vwr.com/nl_NL/pcr_cycler_service.jsp

11. Thermal cycler testing service http://www.isq.pt/EN/services/metrology/\#tab-id-10

12. DRIFTCON Operations Manual http://www.cyclertest.com/Downloads.aspx

13. MTAS Operations Manual http://www.cyclertest.com/Downloads.aspx 